Elsevier required licence: (C) $<2018>$. This manuscript version is made available under the CC-BY-NCND 4.0 license http://creativecommons.org/licenses/by-nc-nd/4.0/

The definitive publisher version is available online at

[https://www.sciencedirect.com/science/article/pii/S00224 60X1830511X?via\%3Dihub] 


\section{Effects of periodically corrugated surfaces on sound scattering}

Shuping Wang ${ }^{\mathrm{a}}$, Jiancheng Tao ${ }^{\mathrm{a},{ }^{*}}, \mathrm{Xiaojun}_{\mathrm{Qiu}}{ }^{\mathrm{b}}$, Jianchun Cheng $^{\mathrm{a}}$

${ }^{1}$ Key Laboratory of Modern Acoustics and Institute of Acoustics, Nanjing University, Nanjing 210093, China

${ }^{2}$ Centre for Audio, Acoustics and Vibration, Faculty of Engineering and IT, University of Technology Sydney, Sydney, NSW 2007, Australia 


\section{ABSTRACT}

Periodic structures have been used in soundscape design to achieve noise control while maintaining aesthetic purposes at the same time. As a kind of periodic structure, periodically corrugated surfaces can work as spatial frequency-selective filters to generate marvelous acoustical phenomena. This paper investigates the effects of periodically corrugated surfaces on sound scattering with an analytical method based on the Kirchhoff-Helmholtz Equation. Three different kinds of corrugated surfaces with periodic right triangle, isosceles triangle and sinusoidal steps are considered. It is found that the fundamental frequency of scattered sound is mainly determined by the period of the surface while the relative amplitude of the harmonics to the fundamental frequency is affected by the step height. Measurements in Mount Maoshan demonstrate that sound scattered from right triangle steps sounds like trumpet solos with the fundamental frequency and all the harmonics of almost the same amplitude. Scattered sound of different timbres can be designed with periodic surfaces of different shapes, which might have potential applications in soundscape design.

Keywords: Sound scattering, Periodic surface, Timbre 


\section{Introduction}

Periodically corrugated surfaces lead to many interesting acoustic phenomena around the world. For example, the El Castillo pyramid produces a sound echo in response to a handclap which sounds like the chirp of Quetzal and observers seated on the lowest step of the great pyramid at Chichen Itza hears pulses that sound like raindrops falling in a water filled bucket when other people are climbing the pyramid higher up [1]. Sound coming from the middle of the Hellenistic theatre reaches the outer seats without too much loss of intensity [2]. Six bugle-like notes are produced as a result of a firecracker being set off in front of the Southern Jiangsu Victory Monument in Mount Maoshan, Jiangsu, China [3].

Much effort has been made to explain these interesting phenomena. The diffraction theory was applied by Declercq et al. to understand the physical effects that cause the formation of the chirp echo in the El Castillo pyramid, but the simulations are only valid when the Lipmann conditions are fulfilled [1]. Fresnel-Kirchhoff's Diffraction theory was used to predict the fundamental frequencies of the six bugle-like notes in Mount Maoshan [3]. The Kirchhoff approximation was applied on the stairs of Kukulkan temple and the simulation results agreed well with the recording [4].

Previous work has also reported the theoretical solution to the scattered sound field caused by periodically corrugated surfaces, for example, the rigorous theory based on the extended boundary condition method proposed by Chuang et al. [5]. In 
1981, Holford proposed an exact solution to the scattered sound from a sinusoidal pressure release boundary [6]. McCammon et al. compared the approximate solutions of Kirchhoff, Eckart, Rayleigh, and Brekovskikh with the exact solution and found that the approximate solutions agreed fairly well with measured data only for low heights and slopes of steps [7]. Chuang et al. proposed a rigorous theory based on the extended boundary condition method, but it can only be applied when the ratio of the height to the period is small [8]. Rayleigh theory is another method to investigate scattering from corrugated surfaces, and it is applied by Purcell to analyze multi-sinusoidal periodic surface [9]. Apart from the theoretical solution, boundary element method is an accurate method to calculate scattered sound fields caused by complex surfaces, but it requires a large number of elements to obtain sufficient accuracy, especially in high frequency range [10-11].

Recently, we proposed a theoretical method based on Kirchhoff-Helmholtz equation to design acoustic landscapes consisting of periodic steps which can transform impulsive sound into trumpet solos [12]. In this paper, the possibility of designing music of different timbres is explored by introducing periodic surfaces of different shapes. The same analytical method was adopted to investigate the effects of periodically corrugated surfaces on the scattered sound field. Three different kinds of corrugated surfaces with periodic right triangle, isosceles triangle and sinusoidal steps are investigated and compared. Both the fundamental frequencies and the amplitudes of the harmonics are discussed and their relationship between the dimensions of the 
steps is explored. Although sinusoidal and triangle-shaped surfaces have been investigated before [13-15], it is the first time that the characteristics of these surfaces are discussed and compared from the aspect of audio effect.

\section{Theory}

Figure 1 shows schematic diagrams of three different kinds of two-dimensional periodic steps: right triangle, isosceles triangle and sinusoidal steps. $N$ steps are located on an infinite rigid baffle ( $y=0$ plane) with the height and length of $H$ and $L$. A point source is located on the right side of the steps above the baffle.
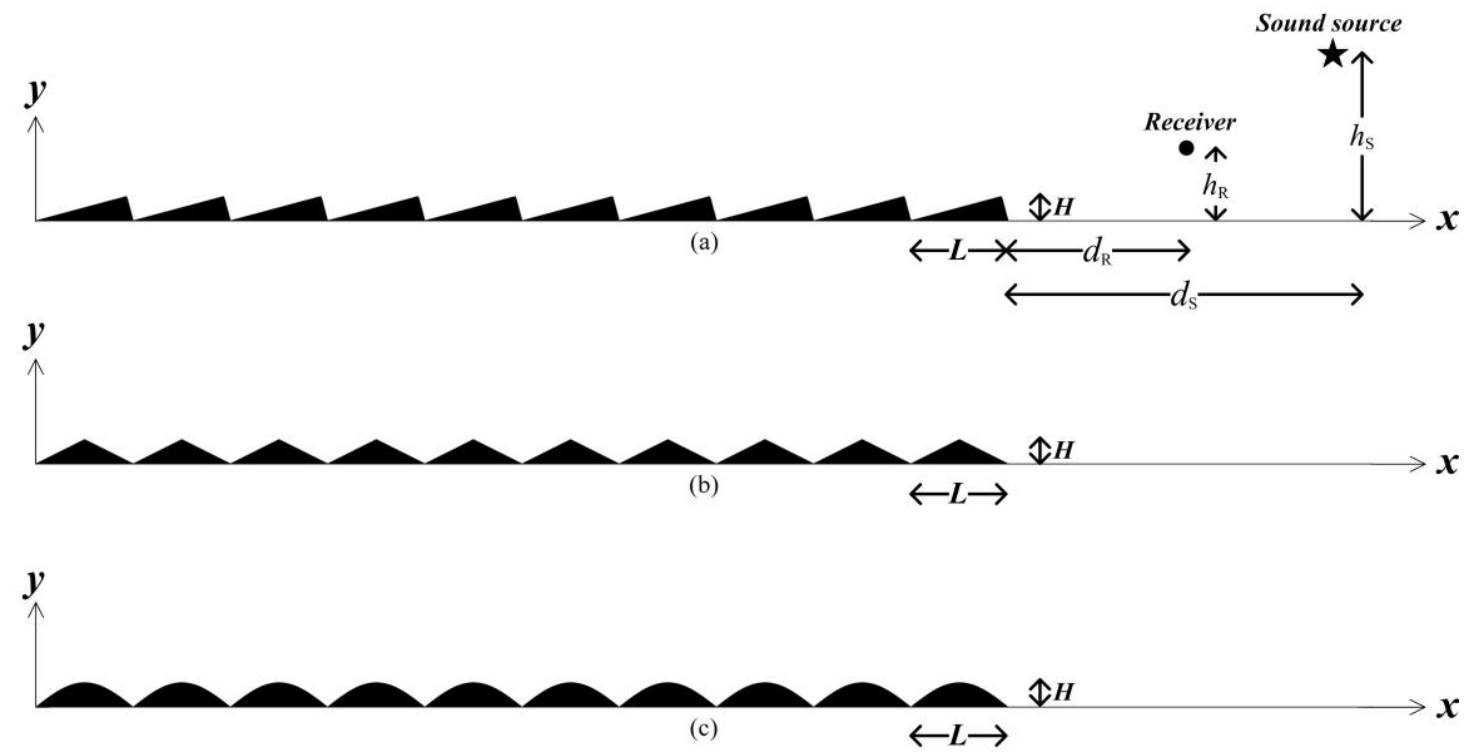

Figure 1: The schematic diagram of two-dimensional periodic steps: (a) right triangle steps, (b) isosceles triangle steps, (c) sinusoidal steps.

The height of the $n$th step in $y$ axis for the right triangle steps in Fig. 1(a) is 


$$
\xi(x)=\left\{\begin{array}{ll}
\frac{H}{D}(x-n L), & n L<x \leq n L+D \\
-\frac{D}{H}[x-(n+1) L], & n L+D<x \leq n L+L
\end{array},\right.
$$

where

$$
D=\frac{\left(L+\sqrt{L^{2}-4 H^{2}}\right)}{2} .
$$

In Fig. 1(b), the height of the $n$th steps in $y$ axis for the isosceles triangle steps is

$$
\xi(x)=\left\{\begin{array}{ll}
\frac{H}{D}(x-n L), & n L<x \leq n L+D \\
-\frac{H}{D}[x-(n+1) L], & n L+D<x \leq n L+L
\end{array},\right.
$$

where $D=L / 2$. The height of the $n$th steps in $y$ axis for the sinusoidal steps in Fig. 1(c) is

$$
\xi(x)=H \sin \left[\frac{\pi}{L}(x-n L)\right], \quad n L<x \leq n L+L .
$$

The scattered sound field is a line integral over the surface of the steps and the baffle [16]

$$
p_{\mathrm{s}}=\int_{\mathrm{A}+\Sigma}\left[G \frac{\partial p}{\partial \boldsymbol{n}}-p \frac{\partial G}{\partial \boldsymbol{n}}\right] d S
$$

where A is the surface of the steps, $\sum$ is the surface of the baffle, $\boldsymbol{n}$ is the normal vector on the surface and $G$ is the Green's function in semi-infinite space (rigid baffle at $y=0)$,

$$
G=-\frac{\mathrm{j}}{4} H_{0}^{(2)}(k r)-\frac{\mathrm{j}}{4} H_{0}^{(2)}\left(k r_{\mathrm{i}}\right)
$$

where $r$ and $r_{\mathrm{i}}$ is the distance from the receiver to the sound source and image source regarding $y=0$ plane, respectively. The surfaces of the steps and baffle are all rigid, 
so Eq. (5) can be expressed as an integral on A [12]

$$
p_{\mathrm{s}}=\int_{\mathrm{A}}-p \frac{\partial G}{\partial \boldsymbol{n}} d S
$$

and further simplified as an integral on $\mathrm{A}^{\prime}$, the projection of A on $x$ axis

$$
p_{\mathrm{s}}=\int_{\mathrm{A}^{\prime}}\left[-p\left(\frac{\partial \xi}{\partial x} \frac{\partial G}{\partial x}-\frac{\partial G}{\partial y}\right)\right] d x
$$

A numerical scheme is used by discretizing the integral function since it is difficult to obtain the analytical solution of Eq. (8). The solution is accurate if the discretization interval is sufficiently small.

\section{Simulations and discussions}

In the simulations, sound speed in the air $c_{0}=343 \mathrm{~m} / \mathrm{s}$, and the density of the air $\rho_{0}=1.225 \mathrm{~kg} / \mathrm{m}^{3}$. The steps in Fig. 1 have the dimensions of $L=0.8 \mathrm{~m}$ and $H=0.1 \mathrm{~m}$ and there are a total of 10 steps distributed from $x=0 \mathrm{~m}$ to $x=8 \mathrm{~m}$. The distance between the source and the steps is $d_{\mathrm{s}}=5.5 \mathrm{~m}$ along $x$ axis, and the distance between the receiver and the steps is $d_{\mathrm{r}}=2.0 \mathrm{~m}$. The height of the sound source and the receiver is $h_{\mathrm{s}}=2.0 \mathrm{~m}$ and $h_{\mathrm{r}}=1.0 \mathrm{~m}$, respectively.

Figure 2(a) shows the scattered sound pressure level (SPL) calculated with the analytical model mentioned in Section 2 and the numerical simulations, which was obtained by creating a model in Ansys 14.5 and importing it into Sysnoise 5.6. It is clear that the theoretical and numerical simulation results agree very well. The peak frequencies appear at around $218 \mathrm{~Hz}$ and its harmonics, which indicates that these two kinds of steps both work as frequency-selective filters. The numerical simulation results for right triangle steps are not included because it has been demonstrated as 
valid in previous work [12].

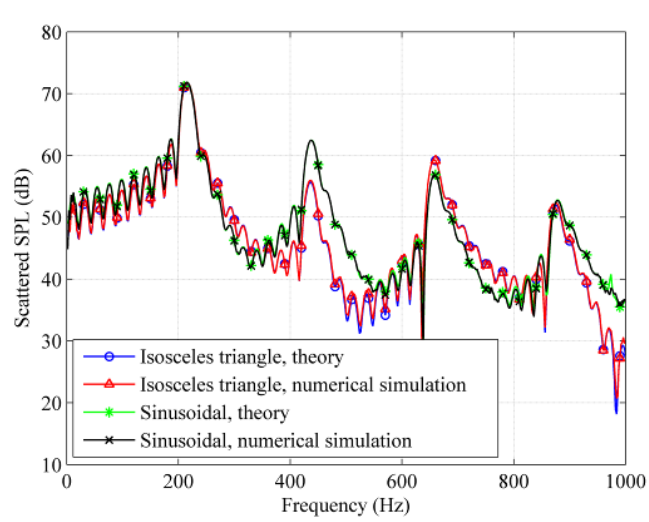

(a)

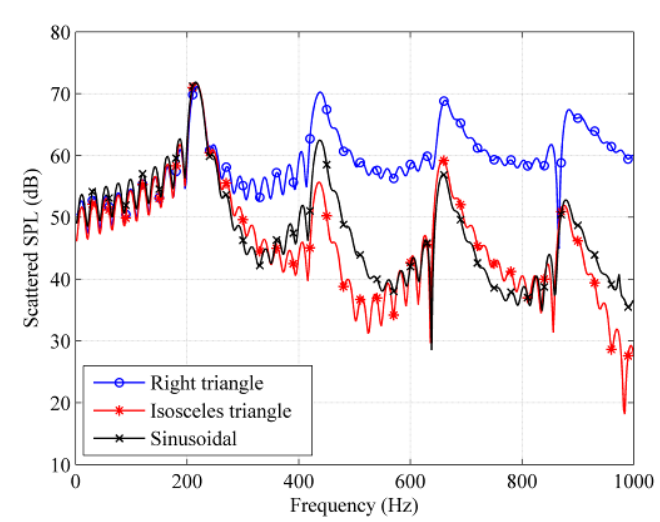

(b)

Figure 2: (a) The scattered sound pressure level (SPL) obtained with the analytical model and numerical simulations, (b) The theoretical scattered sound SPL for three kinds of surfaces calculated with the analytical model, $L=0.8 \mathrm{~m}, H=0.1 \mathrm{~m}, d_{\mathrm{s}}=5.5$ $\mathrm{m}, h_{\mathrm{s}}=2.0 \mathrm{~m}, d_{\mathrm{r}}=2.0 \mathrm{~m}, h_{\mathrm{r}}=1.0 \mathrm{~m}$.

Figure 2(b) shows the theoretical scattered SPL from the three types of surfaces at the same receiver position with the same sound source calculated with the analytical model. All the steps have the dimensions of $L=0.8 \mathrm{~m}$ and $H=0.1 \mathrm{~m}$. It can be seen that the first peak of the scattered sound for the three surfaces are almost the same while the other harmonics have different amplitudes. The amplitudes of all the harmonics are almost the same as that of the fundamental frequency when the steps are right triangles. The amplitudes of peaks decrease with frequencies when the surface is sinusoidal. When the steps are isosceles triangles, the odd harmonics have larger amplitudes than the even harmonics. 
The scattered SPLs at different receiver positions with different sound source positions are shown in Fig. 3. The characteristics of the relative amplitudes of harmonics to the fundamental frequency does not vary with positions of the sound sources and receivers. Another remark is that when the sound source and the receiver are at two different sides of the steps (the squared purple curve, $d_{\mathrm{s}}=8 \mathrm{~m}, h_{\mathrm{s}}=3 \mathrm{~m}, d_{\mathrm{r}}$ $\left.=-12 \mathrm{~m}, h_{\mathrm{r}}=2 \mathrm{~m}\right)$, the frequency selective character cannot be observed any more.

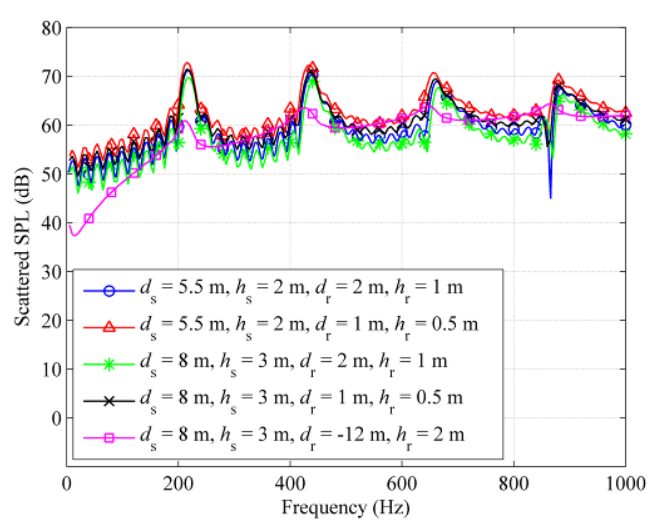

(a)

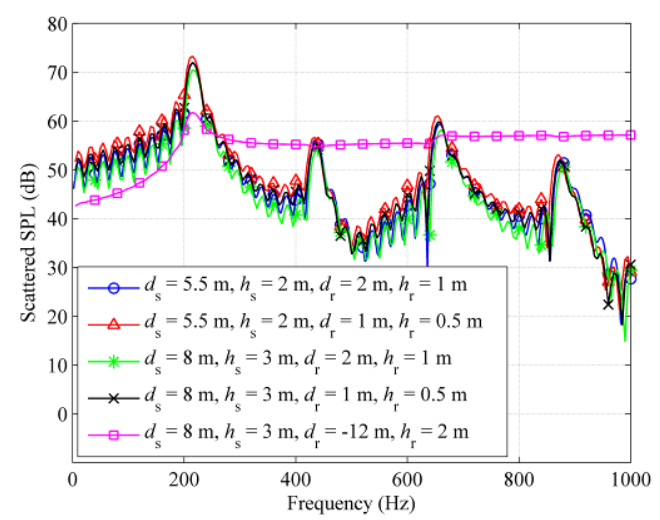

(b)

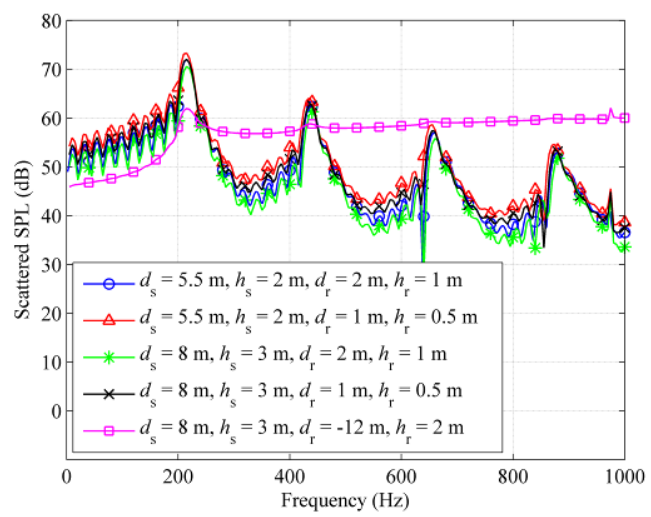

(c)

Figure 3: The scattered SPL with different sound source and receiver positions: (a) right triangle steps, (b) isosceles triangle steps, (c) sinusoidal steps. 
It can also be observed from Fig. 3 that the fundamental frequencies are slightly different at different positions, but the difference is not significant. The relationship between the fundamental frequency and the dimensions of the steps are investigated and the results are shown in Fig. 4, where the position of the sound source is fixed at $d_{\mathrm{s}}=5.5 \mathrm{~m}$ and $h_{\mathrm{s}}=2.0 \mathrm{~m}$, and the receiver is fixed at $d_{\mathrm{r}}=2.0 \mathrm{~m}$ and $h_{\mathrm{r}}=1.0 \mathrm{~m}$. Figures 4(a) and 4(b) indicate that the fundamental frequency decreases with the length (period) of the steps when the height of the steps is fixed as $0.025 \mathrm{~m}$ or $0.05 \mathrm{~m}$. Figures 4(c) and 4(d) show that the fundamental frequency remains almost unchanged with different heights of the steps when the length of the steps is fixed as $0.6 \mathrm{~m}$ or 0.8 $\mathrm{m}$. It is clear that the fundamental frequency is mainly determined by the period of the steps while the effect of the height is small. The fundamental frequency of the scattered sound can be estimated with $f=c_{0} / 2 L$, and it is the same for the three kinds of surfaces if the period of steps is the same.

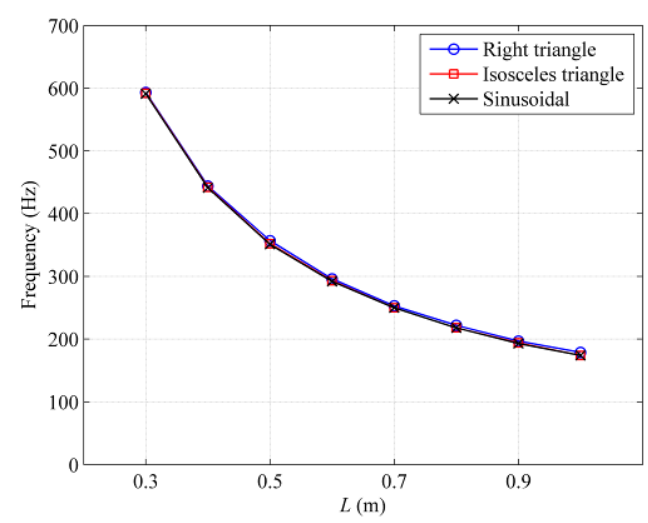

(a)

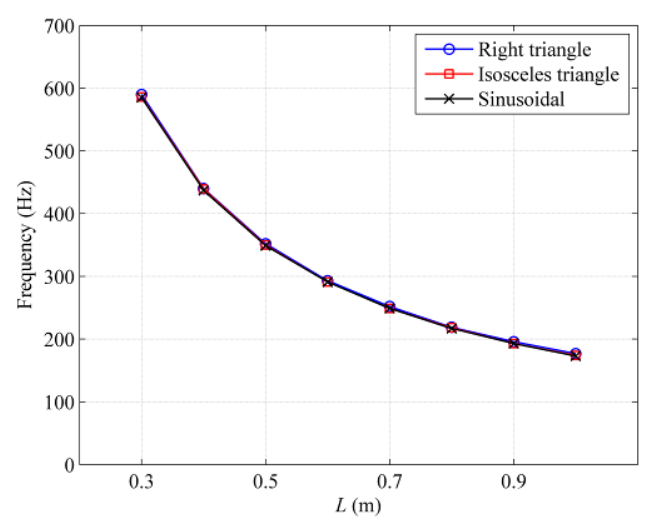

(b) 


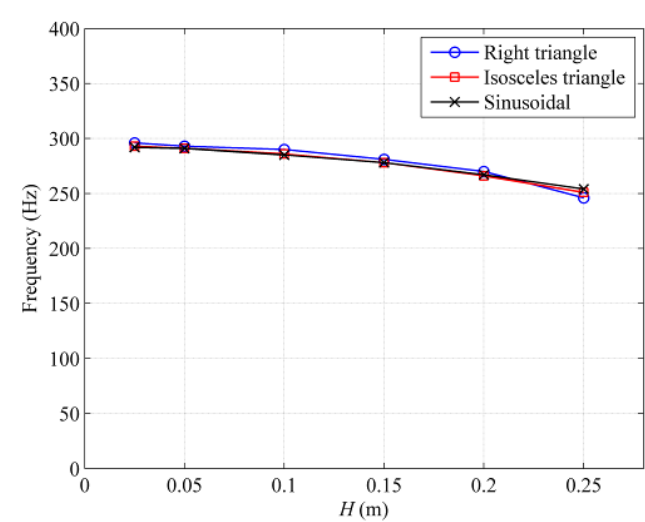

(c)

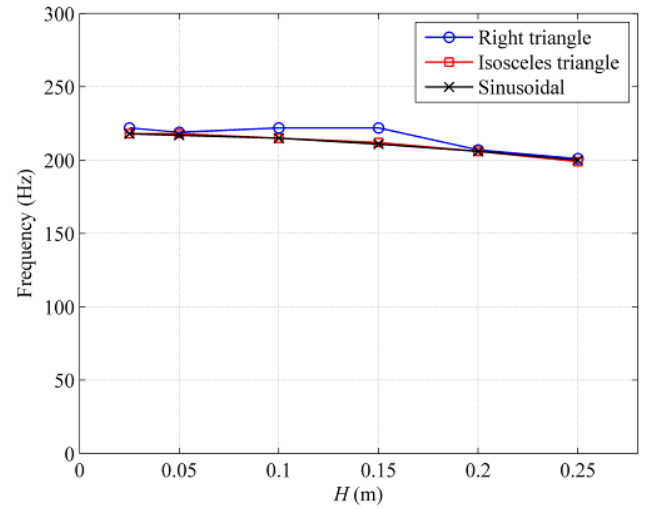

(d)

Figure 4: The fundamental frequencies when the three kinds of periodic steps have different dimensions, $d_{\mathrm{s}}=5.5 \mathrm{~m}, h_{\mathrm{s}}=2 \mathrm{~m}, d_{\mathrm{r}}=2 \mathrm{~m}, h_{\mathrm{r}}=1 \mathrm{~m}$ : (a) $H=0.05 \mathrm{~m}$, (b) $H=$ $0.025 \mathrm{~m}$, (c) $L=0.6 \mathrm{~m}$, (d) $L=0.8 \mathrm{~m}$.

To illustrate the effect of the step height on the scattered sound field, the scattered SPL when the length of the steps $L=0.8 \mathrm{~m}$ is shown in Fig. 5. It can be observed that the scattered SPL increases with the height of the steps. The reason is that the area of the surface increases with $H$. As can be seen from Eq. (7), the scattered sound pressure level is related to the integral of $p$ over the entire surface of the steps, so that the increase of the area will result in the increase of scattered sound pressure. Another observation is that when the steps are isosceles triangles, the amplitudes of the even harmonics increase much faster than the odd harmonics, so that the difference of SPLs between the odd and even harmonics becomes less apparent as the height of steps increases. 


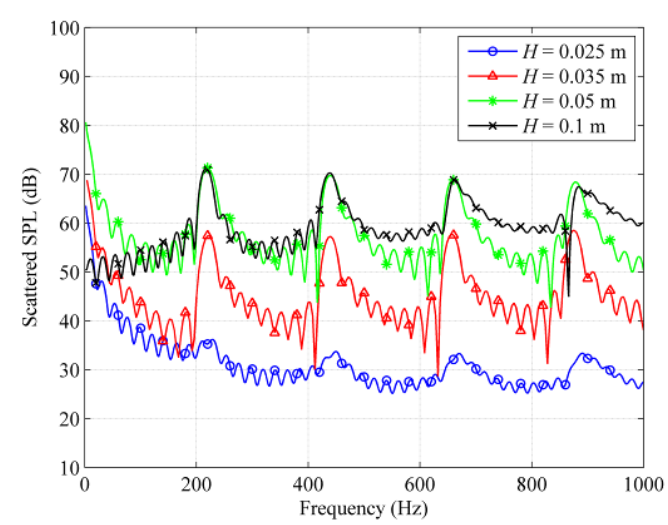

(a)

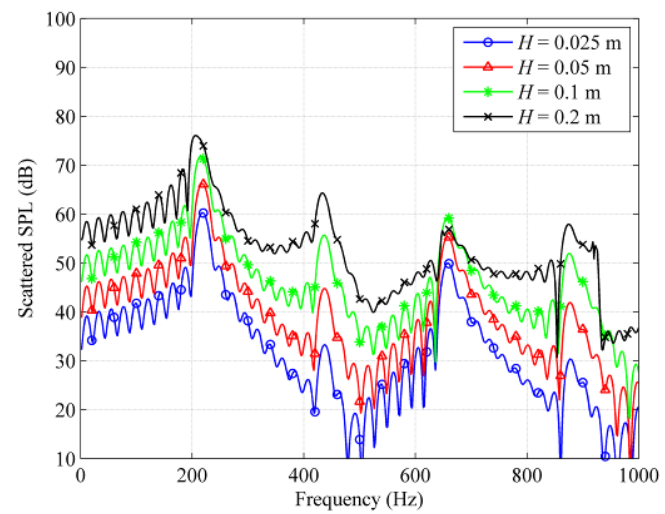

(b)

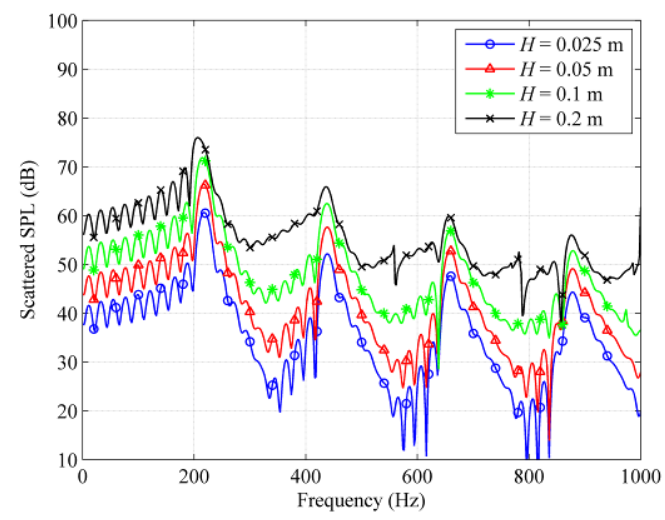

(c)

Figure 5: The scattered SPL when $L=0.8 \mathrm{~m}$ : (a) right triangle steps, (b) isosceles triangle steps, (c) sinusoidal steps.

It can also be seen from Fig. 5 that the peak frequencies, especially the high order harmonics, become less apparent as the height of the steps increases. Figure 6 shows the total sound pressure near the surface of the isosceles triangle and sinusoidal steps with different heights. The frequency of interest is $436 \mathrm{~Hz}$, and the sound source is at $(13.5,2) \mathrm{m}$, which is not shown in these figures. It is clear that the total sound field becomes less uniform as the height of steps increases. When $H=0.2 \mathrm{~m}$, the total 
sound pressure above the 3 or 4 steps nearest to the sound source is much higher than that above the rest of the steps. In this case, the scattered sound pressure is dominated by these 3 or 4 steps, so that the frequency-selective character is not as obvious as the lower steps when all of them work effectively.
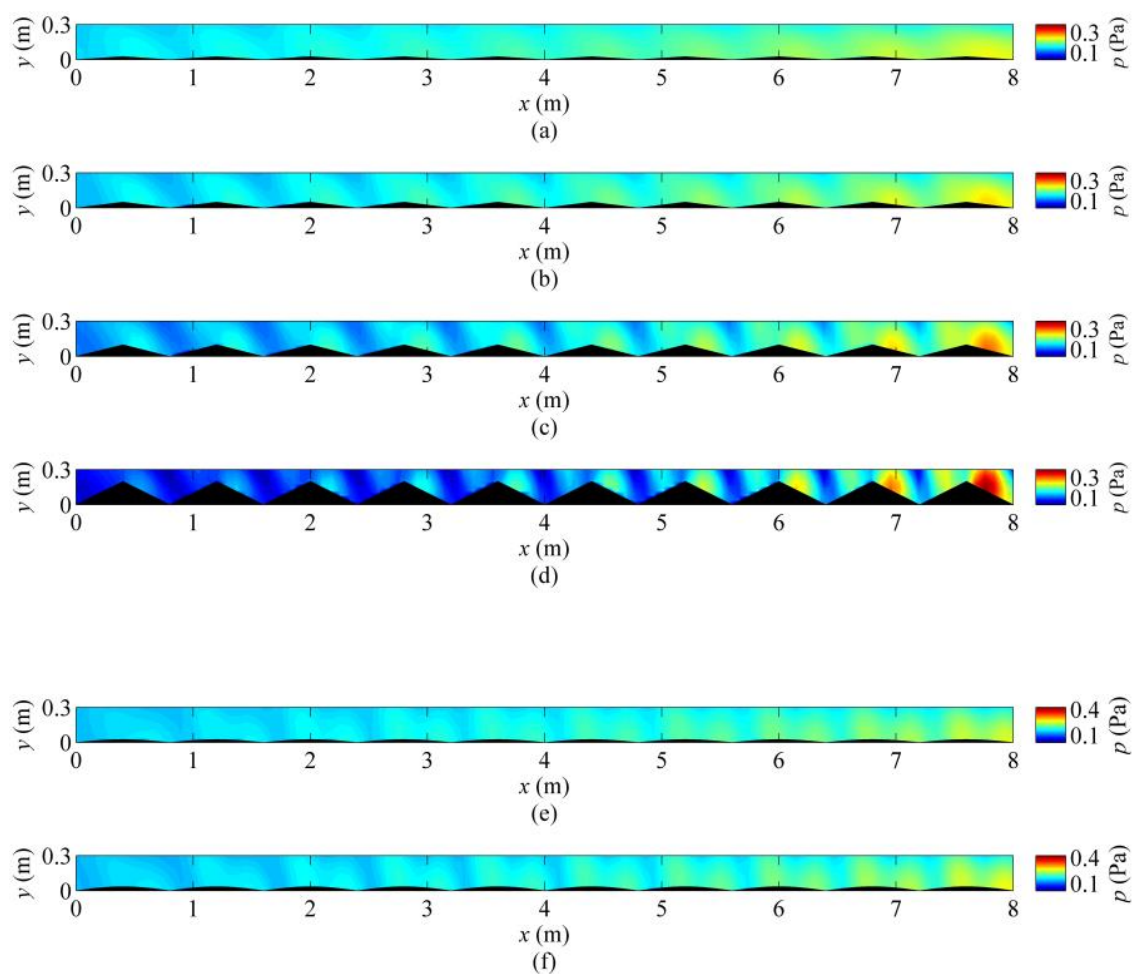

(f)

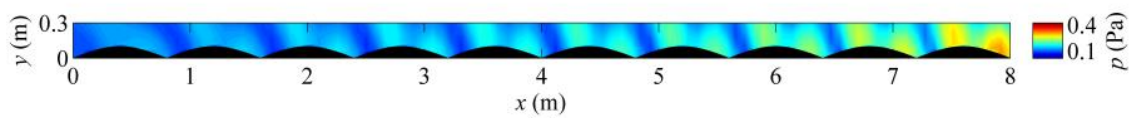

(g)

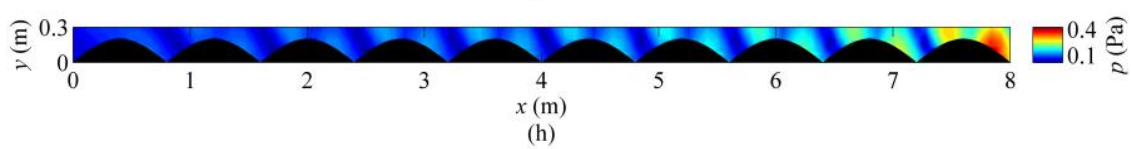

Figure 6: The total sound pressure at $436 \mathrm{~Hz}$ within the area $0 \mathrm{~m}<x<8 \mathrm{~m}, 0 \mathrm{~m}<y<$ $0.3 \mathrm{~m}$, (a) isosceles triangle steps, $H=0.025 \mathrm{~m}$, (b) isosceles triangle steps, $H=0.05$ m, (c) isosceles triangle steps, $H=0.1 \mathrm{~m}$, (d) isosceles triangle steps, $H=0.2 \mathrm{~m}$, (e) sinusoidal steps, $H=0.025 \mathrm{~m}$, (f) sinusoidal steps, $H=0.05 \mathrm{~m}$, (g) sinusoidal steps, $H$ 
$=0.1 \mathrm{~m}$, (h) sinusoidal steps, $H=0.2 \mathrm{~m}$.

Figure 6 also shows that the total sound pressure above the surface is almost the same for the isosceles and sinusoidal steps, so it is the geometry of the surfaces that result in the $6.7 \mathrm{~dB}$ difference of the scattered SPL at $436 \mathrm{~Hz}$ as shown in Fig. 2(b). Since different kinds of surfaces with the same period lead to the same fundamental frequency but different amplitudes of harmonics, it is feasible to design sound of different timbres with a combination of different surfaces. In the design, the desired notes need to be analyzed first in frequency domain to find out the timbre, or the relationship between harmonics and fundamental frequency, so that a proper step shape can be chosen for each note. The length and height of the step is determined by the fundamental frequency and the timbre. More steps in a group result in higher scattered sound pressure level, but the peak frequencies will become less apparent, and the balance between them need to be found in order to decide how many steps are needed in each group.

\section{Measurements in Mount Maoshan}

The measurements were carried out in Mount Maoshan. There are 6 groups of right triangle steps of different sizes, as shown in Fig. 7. The firecracker was set off on the square in front of the steps at the height of $30 \mathrm{~m}$, about $32 \mathrm{~m}$ away from step Group 1. The measurement point is at the height of $1.5 \mathrm{~m}, 30 \mathrm{~m}$ away from Group 1. The recorded signal is shown in Fig. 8(a), in which the red boxes show the 6 echoes 
which are scattered by the 6 groups of steps, respectively.

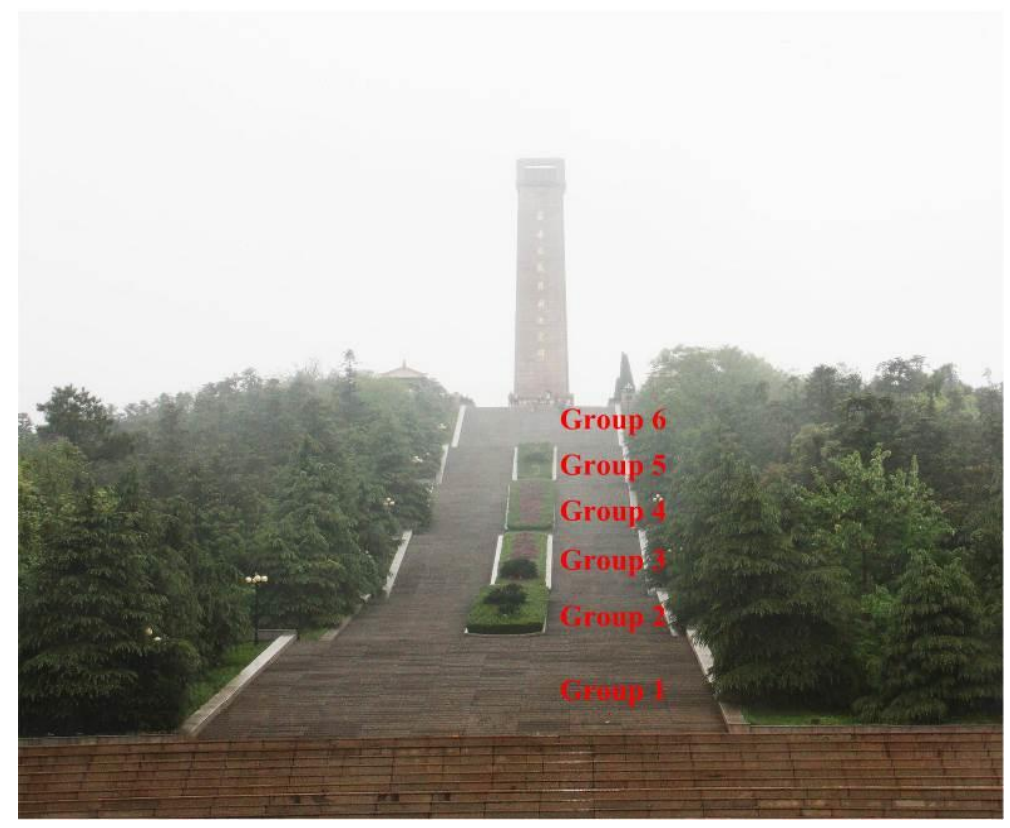

Figure 7: Photo of the 6 groups of steps in Mount Maoshan.

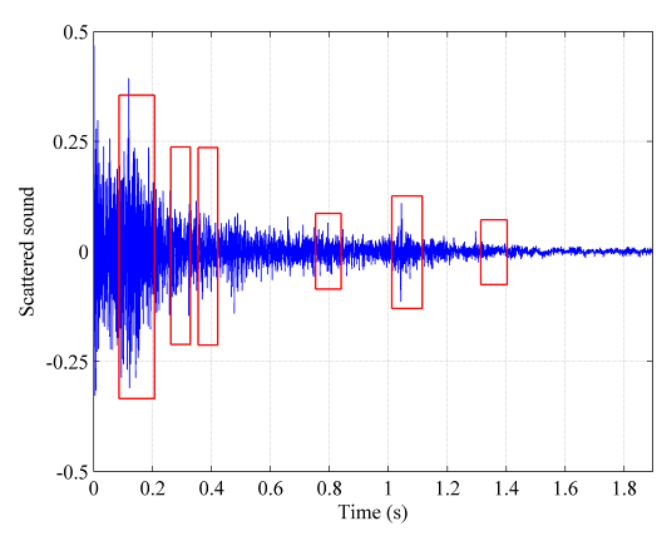

(a)

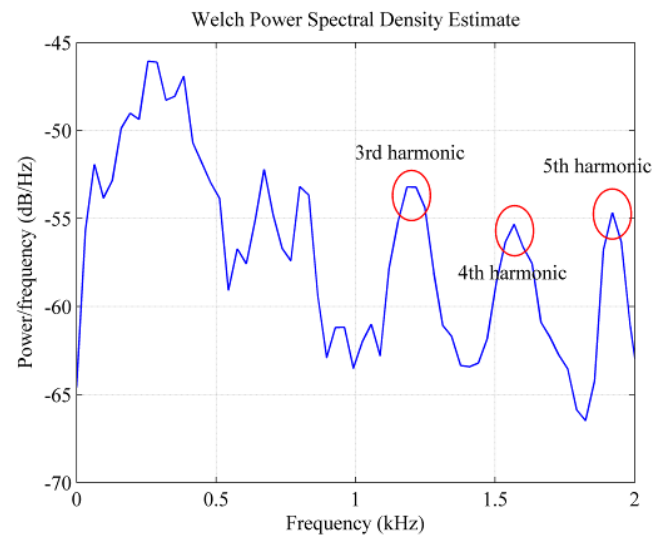

(b)

Figure 8: (a) The recorded time domain signal at Mount Maoshan, (b) The power spectral density of the first echo (sound scattered by steps in Group 1).

Take the first echo as an example. The power spectral density is shown in Fig. 8(b). There are 3 apparent peaks below $2 \mathrm{kHz}$, which correspond to the $3 \mathrm{rd}$, 4th and 
5th harmonics of the fundamental frequency (about $400 \mathrm{~Hz}$ ). Because of the background noise, the first 2 peaks are not very clear. Another observation is that the amplitudes of the 3 peaks are almost the same, which is the characteristics of sound scattered by right triangle steps, as mentioned above in Section 3. The theoretical SPL scattered by 10 steps of the same size as those in Group $1(L=0.53 \mathrm{~m}, H=0.10 \mathrm{~m})$ at the same receiver position is shown in Fig. 9. The calculated fundamental frequency is $408 \mathrm{~Hz}$, which is close to the measurement result (about $400 \mathrm{~Hz}$ ), which further verifies the soundness of the analytical method.

The timbre of music is related to the intensity of harmonics [14]. The 6 echoes in Mount Maoshan phenomenon sound like a trumpet solo because all the harmonics, as well as the fundamental frequency component have similar intensities. As pointed out above, different shapes of steps lead to different amplitudes of the harmonics, which provides the possibility to design music of different timbres and it has promising applications in soundscape design in the future.

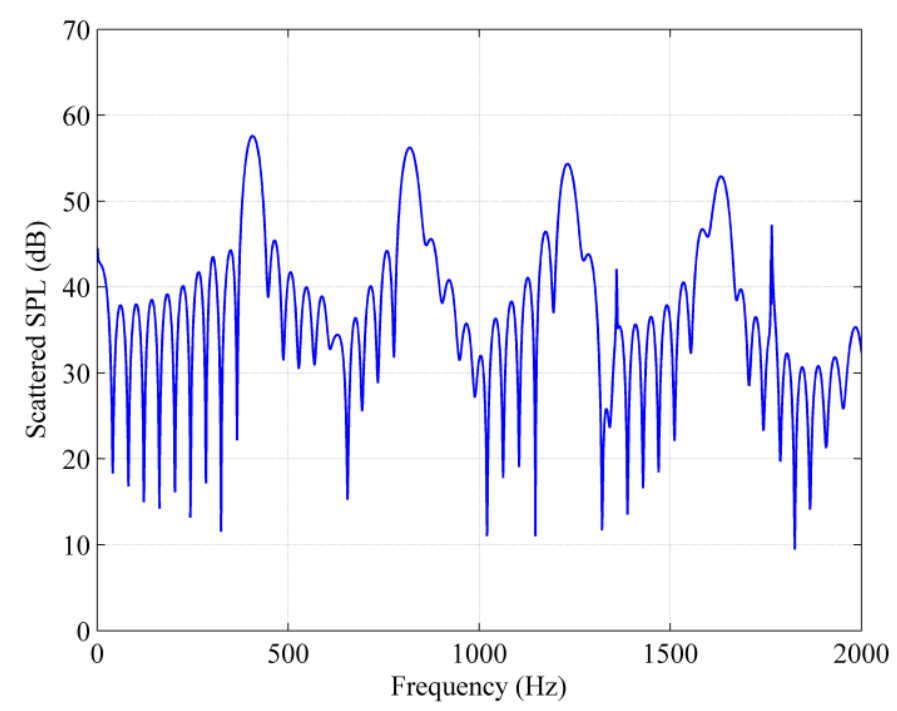

Figure 9: The theoretical scattered SPL of Group 1. 


\section{Conclusions}

An analytical method based on the Kirchhoff-Helmholtz Equation is used to investigate effects of different kinds of periodically corrugated surfaces on timbres of scattered sound. It is found that the fundamental frequency of the scattered sound is mainly determined by the period of the surface while the relative amplitude of the harmonics to the fundamental frequency component is affected by the height of the steps. The periodic right triangle, isosceles triangle and sinusoidal steps with the same height and length lead to the same fundamental frequency, but the amplitudes of harmonics are different, which provides the possibility to design scattered sound of different timbres with a combination of different kinds of surfaces.

\section{Acknowledgements}

This research was supported by the National Natural Science Foundation of China (No. 11474163 and 11634006). 


\section{References}

[1] N. Declercq, J. Degrieck, R. Briers, O. Leroy, A theoretical study of special acoustic effects caused by the staircase of the El Castillo pyramid at the Maya ruins of Chichen-Itza in Mexico, J. Acoust. Soc. Am. 116 (6) (2004) 3328-3335.

[2] N. Declercq, C. Dekeyser, Acoustic diffraction effects at the Hellenistic amphitheater of Epidaurus: Seat rows responsible for the marvelous acoustics, J. Acoust. Soc. Am. 121 (4) (2007) 2011-2022.

[3] X. Chen, M. Qin, S. Wang, H. Zhou, "Unravelling the mystery of the "Maoshan Bugle”, Am. J. Phys. 82 (135) (2014) 135-141.

[4] T. Nicolas, L. Sylvain, D. Carsten, D. Matteo, Extending geometrical acoustics to highly detailed architectural environments, 19th International Congress on Acoustics, Madrid, 2-7 September (2007).

[5] S. Chuang, R. Johnson, Acoustic wave scattering from a fluid/solid periodic rough surface, J. Acoust. Soc. Am. 71 (6) (1982) 1368-1376.

[6] R. Holford, "Scattering of sound waves at a periodic, pressure-release surface: An exact solution,” J. Acoust. Soc. Am. 70(4), 1116-1128, (1981).

[7] D. McCammon and S. McDaniel, "Application of a new theoretical treatment to an old problem; sinusoidal pressure release boundary scattering," J. Acoust. Soc. Am. 78(1), 149-156, (1985).

[8] S. Chuang and R. Johnson, "Acoustic wave scattering from a fluid/solid periodic rough surface," J. Acoust. Soc. Am. 71(6), 1368-1376, (1982). 
[9] A. Purcell, The Rayleigh equations for a multi-sinusoidal periodic surface, J. Acoust. Soc. Am. 103 (2) (1997) 683-694.

[10] M. Karimi, P. Croaker, N. Kessissoglou, Boundary element solution for periodic acoustic problems, J. Sound. Vib. 360 (2016) 129-139.

[11] M. Karimi, P. Croaker, N. Kessissoglou, Acoustic scattering for 3D multi-directional periodic structures using the boundary element method, J. Acoust. Soc. Am. 141 (1) (2017) 313-323.

[12] S. Wang, J. Tao, X. Qiu, J. Cheng, Spatial filtering of audible sound with acoustic landscapes, Appl. Phys. Lett. 111 (2017) 1-5.

[13] R. Holford, Scattering of sound waves at a periodic, pressure-release surface: An exact solution, J. Acoust. Soc. Am. 70 (4) (1981) 1116-1128.

[14] D. McCammon, S. McDaniel, Application of a new theoretical treatment to an old problem; sinusoidal pressure release boundary scattering, J. Acoust. Soc. Am. 78 (1) (1985) 149-156.

[15] A. Jungman, L. Adler, J. Achenbach, R. Roberts, Reflection from a boundary with periodic roughness: Theory and experiment, J. Acoust. Soc. Am. 74 (3) (1983) 1025-1032.

[16] P. Morse, K. Ingard, Theoretical Acoustics, MacMillan, 1986.

[17] H. Fletcher. Loudness, pitch and the timbre of musical tones and their relation to the intensity, the frequency and the overtone structure, J. Acoust. Soc. Am. 6 (59) (1934) 59-69. 